\title{
Weighted coloring: further complexity and approximability results
}

\author{
Bruno Escoffier*, Jérôme Monnot*, Vangelis Th. Paschos*
}

\begin{abstract}
Given a vertex-weighted graph $G=(V, E ; w), w(v) \geq 0$ for any $v \in V$, we consider a weighted version of the coloring problem which consists in finding a partition $\mathcal{S}=\left(S_{1}, \ldots, S_{k}\right)$ of the vertex set $V$ of $G$ into stable sets and minimizing $\sum_{i=1}^{k} w\left(S_{i}\right)$ where the weight of $S$ is defined as $\max \{w(v): v \in S\}$. In this paper, we keep on with the investigation of the complexity and the approximability of this problem by mainly answering one of the questions raised by D. J. Guan and X. Zhu ("A Coloring Problem for Weighted Graphs", Inf. Process. Lett. 61(2):77-81 1997).
\end{abstract}

Key words : Approximation algorithm; NP-complete problems; weighted coloring; interval graphs; line graph of bipartite graphs; partial $k$-tree

\section{Introduction}

A $k$-coloring of $G=(V, E)$ is a partition $\mathcal{S}=\left(S_{1}, \ldots, S_{k}\right)$ of the vertex set $V$ of $G$ into stable sets $S_{i}$. In the usual coloring problem, the objective is to determine a vertex coloring minimizing $k$. A natural generalization of this problem is obtained by assigning a strictly positive integer weight $w(v)$ for any vertex $v \in V$, and defining the weight of stable set $S$ of $G$ as $w(S)=\max \{w(v): v \in S\}$. Then, the objective is to determine a vertex coloring $\mathcal{S}=\left(S_{1}, \ldots, S_{p}\right)$ of $G$ minimizing the quantity $\sum_{i=1}^{p} w\left(S_{i}\right)$. This problem has several applications. For instance in [8] this problem is motivated by a problem of transmission of real-time messages in a metropolitan network or a problem related to dynamic storage allocation. It is interesting to notice that in these two applications, graphs of a special kind are used: the interval graphs. Others examples of applications in different contexts can be found in $[2,5]$.

*LAMSADE, Université Paris-Dauphine, 75775 Paris cedex 16, France. \{escoffier, monnot, paschos\} @lamsade.dauphine.fr 
In this paper, we continue the investigation of the complexity and the approximability of the Weighted Coloring problem by mainly answering one of the questions raised by Guan and Zhu [8].

Given an instance $I=(G, w), W$ denotes the set of different weights used in the instance, i.e., $W=\{w(v): v \in V\}$, opt $(I)$ denotes the weight of an optimal weighted coloring of $I$ and $\chi(I)$ denotes the minimum number of colors used among the optimal weighted colorings of $I$. As indicated in $[4,8]$, this number may be very high, even in trees, although it is always bounded above by $\Delta(G)+1$. Moreover, we can obtain a bound related to the quantities $\chi(G)$ and $|W|$ where $\chi(G)$ is the chromatic number of $G$ and $|W|$ is the number of different weights used in $I$. Precisely, in [4] it is proved that we have $\chi(I) \leq 1+|W|(\chi(G)-1)$ and that this bound is tight for any $q=\chi(G)$ and $r=|W|$ for a family of instances $I_{q, r}$ where the graphs are chordal.

Let's recall some standard definitions about some class of graphs, see [9] for more details. A graph $G$ is chordal iff any cycle $C$ of $G$ of length at least 4 has a chord. There are several characterizations of chordal graphs; one of them uses the notion of perfect elimination order (peo. in short) of $G=(V, E)$. An order $v_{1}, \ldots, v_{n}$ of the vertex set $V$ is a peo. if the neighborhood of $v_{i}$ in the subgraph induced by $\left\{v_{1}, \ldots, v_{i}\right\}$ is a clique; a graph is chordal iff it has a peo. When a graph $G$ has a peo., we can easily find an optimal coloring of $G$ by applying the greedy algorithm (take recursively a vertex not colored yet and color it with the smallest color) following the peo. of the vertices. A graph $G=(V, E)$ is a split graph iff one can partition $V$ into $V_{1}, V_{2}$ such that $V_{1}$ is a stable and $V_{2}$ is a clique (there may be some edges linking $V_{1}$ to $V_{2}$ ). A split graph is in particular a chordal graph since it has a peo. A graph is a $k$-tree iff $G$ has a peo. $v_{1}, \ldots, v_{n}$ such that $v_{1}, \ldots, v_{k}$ is a clique and the neighborhood of $v_{i}$ in the subgraph induced by $\left\{v_{1}, \ldots, v_{i}\right\}$ has a size $k$ for $i>k$ (a 1-tree is also called a tree). A graph $G$ is a partial $k$-tree if $G$ is a partial graph of a $k$-tree. A graph $G$ is a comparability graph iff $G$ has a direct orientation $\vec{G}=(V, \vec{E})$ such that $\vec{G}$ is acyclic (there is no circuit) and $\vec{G}$ is transitive (that is if $(x, y) \in \vec{E}$ and $(y, z) \in \vec{E}$, then $(x, z) \in \vec{E})$. A direct acyclic transitive orientation of $G$ is also called a poset. As previously, we can prove that the coloring problem is polynomial in posets. A graph $G$ is a co-comparability graph iff the complement of $G$ is a comparability graph. A graph $G=(V, E)$ is an interval graph if it is the intersection graph of a family of open intervals. For graph-theoretical terms not defined here, the reader is referred to [1].

This paper is organized as follows. In Sect. 2, we answer a question raised in [8] by proving that Weighted Coloring is (strongly) NP-hard in interval graphs. In Sect. 3, we deal with polynomial approximation of Weighted Coloring, providing mainly approximation algorithms for graphs colorable with a few number of colors and for partial $k$-trees. 


\section{Interval graphs}

The interval graphs are a kind of graphs very used in practice, in particular when dealing with scheduling problems. A well known characterization of interval graphs is the following: $G$ is an interval graph iff $G$ is a chordal graph and $G$ is a co-comparability graph. Although the coloring problem is polynomial in interval graphs (since an interval graph is a chordal graph), in [4], it is proved that the Weighted Coloring problem is strongly NPhard in split graphs and thus strongly NP-hard in chordal graphs, since the split graphs is a subclass of chordal graphs. Moreover, it is shown in [5] that the Weighted Coloring problem is polynomial in complements of interval graphs. In this section, we prove that the Weighted Coloring problem is strongly NP-hard in interval graphs.

Theorem 1 Weighted Coloring is strongly NP-hard in interval graphs. Moreover, the problem of finding $\chi(G, w)$ is also NP-hard in interval graphs.

Proof. We reduce the Circular Arc Coloring problem to our problem. A circular arc graph is the intersection graph of arcs of a circle. Garey et al. [6] proved that the Circular Arc Coloring problem, i.e., the problem of finding a minimum size coloring in circular arc graphs, is NP-complete. Let $G$ be the intersection graph of the $n$-tuple of circular arcs $\mathcal{A}=\left(A_{j}\right)_{j \in\{1, \cdots, n\}}$, and let $k \in\{1, \cdots, n\}$. Assume, wlog., that the intervals $A_{j}$ are open. We transform this instance of Circular Arc Coloring in an instance $I^{\prime}=\left(G^{\prime}, w\right)$ of Weighted Coloring as follows. Let $a$ be any point on the circle, and $J_{0}=\left\{j: a \in A_{j}\right\}$. For simplicity, assume wlog. that point $a$ belongs to some arcs and that $J_{0}=\left\{1, \cdots, j_{0}\right\}$, for some $j_{0} \geq 1$. For any $j \leq j_{0}$, we split interval $A_{j}=\left(c_{j}, d_{j}\right)$ in $A_{j}^{\prime}=\left(c_{j}, a\right)$ and $A_{j}^{\prime \prime}=\left(a, d_{j}\right)$. For $j>j_{0}$, we define $A_{j}^{\prime}=A_{j}$. Set $\mathcal{A}^{\prime}$ be the $\left(n+j_{0}\right)$-tuple of intervals $\left(A_{j}^{\prime}\right)_{j \in\{1, \cdots, n\}}$ and $\left(A_{j}^{\prime \prime}\right)_{j \leq j_{0}}$. Let $G^{\prime}$ be the intersection graphs of $\mathcal{A}^{\prime}$. We set the weights $w$ of $G^{\prime}$ in the following way: $w\left(v_{j}^{\prime}\right)=w\left(v_{j}^{\prime \prime}\right)=2 k\left(j_{0}+1-j\right)$ if $j \leq j_{0}$ and $w\left(v_{j}^{\prime}\right)=1$ for $j>j_{0}$. The description of instance $I^{\prime}=\left(G^{\prime}, w^{\prime}\right)$ of Weighted Coloring is now complete.

Note that $\left\{v_{j}, j \leq j_{0}\right\}$ is a clique in $\mathrm{G}$. We can suppose $k \geq j_{0}$ (otherwise $G$ is trivially not $k$-colorable). We claim that $\chi(G) \leq k$ if and only if $\operatorname{opt}\left(G^{\prime}\right) \leq k j_{0}\left(j_{0}+1\right)+k-j_{0}=$ $B$.

Suppose that $\mathcal{S}=\left(S_{1}, \cdots, S_{k}\right)$ is a coloring of $G$. Then, set $\mathcal{S}^{\prime}=\left(S_{1}^{\prime}, \cdots, S_{k}^{\prime}\right)$ where $S_{i}^{\prime}=S_{i} \backslash\left\{v_{j}: j \leq j_{0}\right\} \cup\left\{v_{j}^{\prime}, v_{j}^{\prime \prime}: v_{j} \in S_{i}, j \leq j_{0}\right\}$. One can easily see that $\mathcal{S}^{\prime}$ is a coloring of $G^{\prime}$. Furthermore, we have $\operatorname{opt}\left(G^{\prime}\right) \leq w\left(\mathcal{S}^{\prime}\right)=2 k \sum_{j=1}^{j_{0}} j+\left(k-j_{0}\right)=B$.

Reciprocally, let $\mathcal{S}^{\prime}=\left(S_{1}^{\prime}, \cdots, S_{l}^{\prime}\right)$ be a coloring of $G^{\prime}$ with $\operatorname{opt}\left(I^{\prime}\right)=w\left(\mathcal{S}^{\prime}\right) \leq$ $B$. Assume that $w\left(S_{i}\right) \geq w\left(S_{j}\right)$ for any $j \geq i$. Note that $\left\{v_{1}^{\prime}, v_{1}^{\prime \prime}\right\} \in S_{1}^{\prime}$, otherwise $\operatorname{opt}\left(I^{\prime}\right)=w\left(\mathcal{S}^{\prime}\right) \geq 2 k j_{0}+2 k j_{0}+2 k \sum_{j=1}^{j_{0}-2} j=k j_{0}\left(j_{0}+1\right)+2 k j_{0}>B$. With an analogous argument, we can show that $\left\{v_{j}^{\prime}, v_{j}^{\prime \prime}\right\} \in S_{j}^{\prime}$ for any $j \leq j_{0}$. Consequently, $w\left(\mathcal{S}^{\prime}\right)=k j_{0}\left(j_{0}+1\right)+\left(l-j_{0}\right)$, and then $l \leq k$. Set $S_{i}=S_{i}^{\prime} \backslash\left\{v_{i}^{\prime}, v_{i}^{\prime \prime}\right\} \cup v_{i}$ for $i \leq j_{0}$ and $S_{i}=S_{i}^{\prime}$ for $i>j_{0} . \mathcal{S}=\left(S_{1} ; \cdots, S_{l}\right)$ is a $l$-coloring of $G$, and $\chi(G) \leq l \leq k$. 
The NP-hardness of computing $\chi\left(I^{\prime}\right)$ follows easily from the previous proof. Indeed, if $G^{\prime}$ is not $k$-colorable, then obviously $\chi\left(I^{\prime}\right)>k$. Otherwise, let $\mathcal{S}^{\prime}$ be an optimal coloring. Since $\chi(G) \leq k$ iff $o p t\left(I^{\prime}\right) \leq B, w\left(\mathcal{S}^{\prime}\right) \leq B$ and, as we have seen above, $w\left(S^{\prime}\right)=k j_{0}\left(j_{0}+1\right)+\left|\mathcal{S}^{\prime}\right|-j_{0}=B+\left|\mathcal{S}^{\prime}\right|-k$. Hence, $\chi\left(I^{\prime}\right) \leq\left|\mathcal{S}^{\prime}\right| \leq k$.

Using Theorem 1 and the characterization of interval graphs, we deduce that the Weighted Coloring problem is strongly NP-hard in co-comparability graphs.

\section{Approximation results}

\section{$3.1 k$-colorable graphs}

We study in this section, the approximability of the Weighted Coloring problem in natural classes of graphs colorable with a few number of colors. We first focus ourselves on subfamilies of $k$-colorable graphs where the minimum coloring problem is polynomial. Our objective is to prove the following theorem.

Theorem 2 Let $\mathcal{G}$ be a class of $k$-colorable graphs, where a $k$-coloring is computable in polynomial time. Then, in any $G \in \mathcal{G}$, Weighted Coloring is approximable within ratio $k^{3} /\left(3 k^{2}-3 k+1\right)$.

Proof. Consider some graph $G=(V, E) \in \mathcal{G}$ of order $n$, and assume that any $v_{i} \in V$ has weight $w_{i}=w\left(v_{i}\right)$. Suppose that $w_{1} \geq w_{2} \geq \cdots \geq w_{n}$. Consider an optimal weighted coloring $\mathcal{S}^{*}=\left(S_{1}^{*}, \cdots, S_{l}^{*}\right)$, with $w\left(S_{1}^{*}\right) \geq \cdots \geq w\left(S_{l}^{*}\right)$ and denote by $i_{k}^{*}$, the index of the heaviest vertex in color $S_{k}^{*}$ (hence, $w\left(S_{k}^{*}\right)=w_{i_{k}^{*}}$ ), by $V_{i}$ the set of vertices $\left\{v_{1}, \cdots, v_{i}\right\}$ (hence, $V_{n}=V$ ) and by $G\left[V^{\prime}\right]$ the subgraph of $G$ induced by $V^{\prime} \subseteq V$.

We compute several colorings of $G$ and choose as final solution the best one among the colorings computed. We first compute a $k$-coloring $\mathcal{S}^{0}$ of $G$. Clearly:

$$
w\left(\mathcal{S}^{0}\right) \leq k w_{1}=k w\left(S_{1}^{*}\right)
$$

Then, for $j=2, \cdots, n+1$, we do the following:

- if $G\left[V_{j-1}\right]$ is bipartite then:

- consider the best weighted 2-coloring $\left(S_{1}^{j}, S_{2}^{j}\right)$ among the 2-colorings of $G\left[V_{j-1}\right]$ ( $S_{2}^{j}$ may be empty);

- color the remaining vertices $v_{j}, \cdots, v_{n}$ with $k$ colors $\left(S_{3}^{j}, S_{4}^{j}, \cdots, S_{k+2}^{j}\right)$, thus obtaining a coloring $\mathcal{S}^{j}=\left(S_{1}^{j}, S_{2}^{j}, \cdots, S_{k+2}^{j}\right)$ of $G$. 
Note that the first step is easily polynomially computable (merge optimally the unique 2-colorings of any connected component).

Consider now the iterations where $j=i_{2}^{*}$ and $j=i_{3}^{*}$. For $j=i_{2}^{*}, V_{j-1}$ is an independent set; hence, $S_{1}^{j}=V_{j-1}$. We get in this case:

$$
w\left(\mathcal{S}^{j}\right) \leq w_{1}+k w_{j}=w\left(S_{1}^{*}\right)+k w\left(S_{2}^{*}\right)
$$

On the other hand, for $j=i_{3}^{*}, G\left[V_{j-1}\right]$ is bipartite; hence, $w\left(S_{1}^{j}\right)+w\left(S_{2}^{j}\right) \leq w\left(S_{1}^{*}\right)+$ $w\left(S_{2}^{*}\right)$. In this case:

$$
w\left(\mathcal{S}^{j}\right) \leq w\left(S_{1}^{*}\right)+w\left(S_{2}^{*}\right)+k w\left(S_{3}^{*}\right)
$$

Recall that the algorithm returns the best coloring among those computed. Note also that if the number $l$ of colors in $\mathcal{S}^{*}$ is smaller than 2, then this algorithm computes an optimal coloring. Combination of equations (3.1), (3.2) and (3.3) with coefficients $(k-1)^{2} / k^{3}$, $k(k-1) / k^{3}$ and $k^{2} / k^{3}=1 / k$, respectively, concludes that the output coloring $\mathcal{S}$ is such that: $w(\mathcal{S}) \leq\left(k^{3} /\left(3 k^{2}-3 k+1\right)\right) w\left(\mathcal{S}^{*}\right)$ and the result follows.

Note that this improves a $(4-3 / k)$-approximation algorithm given in [15] (see the note on related works at the end of the paper), for $k \leq 10$.

Corollary 1 Weighted Coloring is approximable within ratio $27 / 19<1.42$ in polynomially 3-colorable graphs.

It is well known that the coloring problem is polynomial in planar triangle-free graphs ([10]) and that the chromatic number in these graphs is bounded by 3. Moreover, it is proved in [3] that on the one hand the Weighted Coloring problem is strongly NP-hard and, on the other hand, the Weighted Coloring problem cannot be approximated with performance ratio better than $\frac{7}{6}-\varepsilon$ for any $\varepsilon>0$ unless $\mathbf{P} \neq \mathbf{N P}$, in the planar triangle-free graphs, even if the maximum degree is bounded by 4 . Using Theorem 2, we obtain:

Corollary 2 Weighted Coloring is 27/19-approximable in planar triangle-free graphs.

As another corollary of Theorem 2, Weighted Coloring is approximable within ratio 64/37 in polynomially 4-colorable graphs. On the other hand, note that minimum coloring is not $(4 / 3-\varepsilon)$-approximable in planar graphs, that these graphs are polynomially 4-colorable and that the Weighted Coloring problem is a generalization of the coloring problem. Putting all this together, we obtain:

Theorem 3 Weighted Coloring is approximable within ratio 64/37< 1.73 in planar graphs, but it is not $(4 / 3-\varepsilon)$-approximable in these graphs. 
Note that the result of Theorem 2 can be applied also to line graphs of bipartite graphs of degree at most $\Delta$. A weighted coloring on $I=(L(G), w)$ where $L(G)$ is the line graph of $G$ can be viewed as a weighted edge-coloring on $(G, w)$. In fact, in [4], it is shown that the Weighted Coloring problem is strongly NP-complete in line graphs of regular bipartite graphs of degree $\Delta$ and that the Weighted Coloring problem is not $\left(\frac{2^{\Delta}}{2^{\Delta}-1}-\varepsilon\right)$ approximable unless $\mathbf{P}=\mathbf{N P}$, for any $\Delta \geq 3$. Besides, the $\mathbf{N P}$-completeness also holds for the line graphs of complete bipartite graphs. More recently, in [3] this lower bound is tightened up to $\frac{7}{6}$ when $\Delta=3$. Furthermore, it is proved that this bound is the best possible since in [3] is also provided a $7 / 6$-approximation algorithm.

Now, we generalize the technique used in Theorem 2 to get an approximation algorithm in line graphs of bipartite graphs of degree at most $\Delta$, for any fixed $\Delta \geq 3$, since using König's theorem ([12]) we know that the coloring problem is polynomial in linegraphs of bipartite graphs. More precisely, we can show the following theorem:

Theorem 4 For any $\Delta \geq 3$, Weighted Coloring in line graphs of bipartite graphs of maximum degree at most $\Delta$ is approximable within approximation ratio $\rho_{\Delta}$, where $\rho_{1}=$ $\rho_{2}=1$ and:

$$
\rho_{\Delta}=\frac{\Delta}{\sum_{j=1}^{\Delta} \prod_{l=j}^{\Delta-1}\left(1-\rho_{l} / \Delta\right)}
$$

Proof. Let $\rho_{1}=\rho_{2}=1$ and $\rho_{j}$ be a ratio guaranteed by some polynomial algorithm for $3 \leq j \leq \Delta-1$ on line graphs of bipartite graphs of maximum degree at most $j$. Then, on an instance $I=(G, w)$ where $G=L(H)$ and $H$ is a bipartite graph of maximum degree $\Delta$. Let us consider an optimal solution $\mathcal{S}^{*}=\left(S_{1}^{*}, \cdots, S_{l}^{*}\right)$. We have $l \geq \Delta$ by construction. As previously, set $i_{k}^{*}$ the index of the heaviest vertex of $S_{k}^{*}$. Then, as in the proof of Theorem 2, we can compute three colorings of value at most $\Delta w\left(S_{1}^{*}\right)$, $w\left(S_{1}^{*}\right)+\Delta w\left(S_{2}^{*}\right)$ and $w\left(S_{1}^{*}\right)+w\left(S_{2}^{*}\right)+\Delta w\left(S_{3}^{*}\right)$, respectively. Moreover, for any $k \in$ $\{4, \cdots, \Delta\}$, consider the graph $G\left[V_{i_{k}^{*}-1}\right]$ induced by the vertices $V_{i_{k}^{*}-1}=\left\{v_{1}, \cdots, v_{i_{k}^{*}-1}\right\}$. This graph is a line graph of a bipartite graph of maximum degree at most $k-1$ (since it is $k-1$ colorable). Hence, by applying our approximation algorithm with ratio $\rho_{k-1}$ on $I_{i_{k}^{*}-1}=\left(G\left[V_{i_{k}^{*}-1}\right], w\right)$, we can get a coloring of $I_{i_{k}^{*}-1}$ of value at most $\rho_{k-1} \operatorname{opt}\left(I_{i_{k}^{*}-1}\right) \leq$ $\rho_{k-1} \sum_{j=1}^{k-1} w\left(S_{j}^{*}\right)$ and then, a coloring of $I$ of value at most $\rho_{k-1} \sum_{j=1}^{k-1} w\left(S_{j}^{*}\right)+\Delta \times$ $w\left(S_{k}^{*}\right)$. If we take the best coloring $\mathcal{S}$ among all these colorings, we get:

$$
\begin{aligned}
w(\mathcal{S}) & \leq \Delta \times w\left(S_{1}^{*}\right) \\
w(\mathcal{S}) & \leq \rho_{1} w\left(S_{1}^{*}\right)+\Delta \times w\left(S_{2}^{*}\right) \\
& \cdots \\
w(\mathcal{S}) & \leq \rho_{\Delta-1} \sum_{m=1}^{\Delta-1} w\left(S_{m}^{*}\right)+\Delta \times w\left(S_{\Delta}^{*}\right)
\end{aligned}
$$


Let $\beta=\sum_{j=1}^{\Delta} \prod_{l=j}^{\Delta-1}\left(1-\rho_{l} / \Delta\right)$ (note that by convention $\prod_{l=\Delta}^{\Delta-1}\left(1-\rho_{l} / \Delta\right)=1$ ). Then, take the convex combination of these $\Delta$ inequalities with coefficients $\alpha_{1}, \cdots, \alpha_{\Delta}$, where $\alpha_{i}=\frac{\prod_{l=i}^{\Delta-1}\left(1-\rho_{l} / \Delta\right)}{\beta}$. We have $\alpha_{i} \in[0,1], \alpha_{\Delta}=\frac{1}{\beta}$ and $\sum_{i=1}^{\Delta} \alpha_{i}=1$.

We get $w(\mathcal{S}) \leq \rho_{\Delta}\left(\sum_{m=1}^{\Delta} w\left(S_{m}^{*}\right)\right) \leq \rho_{\Delta} w\left(S^{*}\right)$ where $\rho_{\Delta}=\Delta / \beta$. Indeed, the contribution of weight $w\left(S_{i}^{*}\right)$ in the convex combination is $\Delta \alpha_{i}+\sum_{j=i+1}^{\Delta} \alpha_{j} \times \rho_{j-1}$. If we denote $A_{i}=\sum_{j=i+1}^{\Delta} \alpha_{j} \times \rho_{j-1}$, then we can easily prove that $A_{i}=\Delta\left(\alpha_{\Delta}-\alpha_{i}\right)$.

The following table gives the approximate value of ratio $\rho_{\Delta}$ for some $\Delta$.

\begin{tabular}{|c|c|c|c|c|c|c|c|c|c|}
\hline$\Delta$ & 4 & 5 & 10 & 50 & 100 & 200 & 400 & 700 & 1000 \\
\hline$\rho_{\Delta}$ & 1.61 & 1.75 & 2.16 & 2.97 & 3.25 & 3.51 & 3.73 & 3.89 & 3.99 \\
\hline
\end{tabular}

Note that this improves a $(4-3 / \Delta)$-approximation algorithm given in [15] (see the note on related works at the end of the paper), for $\Delta \leq 1025$.

We end this section by improving the lower bound obtained in [4] for the Weighted Coloring problem in line graphs of regular bipartite graphs of degree $\Delta$.

Theorem 5 For any $\Delta \geq 3, \varepsilon>0$, the Weighted Coloring problem is not $\left(1+\frac{2}{\Delta}-\frac{2}{\Delta+1}-\varepsilon\right)$ approximable unless $\boldsymbol{P}=\mathbf{N P}$, in line graphs of regular bipartite graphs of degree $\Delta$.

Proof. For simplicity, we consider the edge model, i.e., we study the Weighted EdgeColoring problem in regular bipartite graphs of degree $\Delta$. We prove the following result by induction: it is NP-complete to distinguish between $\operatorname{opt}(I) \leq \frac{\Delta(\Delta+1)}{2}$ and $\operatorname{opt}(I) \geq$ $\frac{\Delta(\Delta+1)+2}{2}$ in regular bipartite graphs of degree $\Delta$, where the weights used are in $W=$ $\{1, \ldots, \Delta\}$ and there exists at least one vertex of degree $\Delta$ whose incident edges have different weights (i.e. are weighted by $1,2, \ldots$, and $\Delta$ ).

For $\Delta=3$ the result is proved in [3]. Assume that the result holds for $\Delta=k-1$ and let us prove the result for $\Delta^{\prime}=k$.

Let $I=(G, w)$ be an instance with $\Delta=k-1$ (in other words, $G=(L, R ; E)$ is a regular bipartite graphs of degree $k-1)$. We construct an instance $I^{\prime}=\left(G^{\prime}, w^{\prime}\right)$ of the case $\Delta^{\prime}=k$ as follows: we duplicate $G$ as $G_{1}=\left(L_{1}, R_{1} ; E_{1}\right)$ and $G_{2}=\left(L_{2}, R_{2} ; E_{2}\right)$ and we add two matchings $M_{1}$ and $M_{2}$ between $G_{1}$ and $G_{2}$ such that $M_{i}$ links the vertices of $L_{i}$ to the vertices of $R_{3-i}$. The weights of the edges of $G^{\prime}$ are assigned as follows: if $e \in E_{1} \cup E_{2}$, then $w^{\prime}(e)=w(e)$; if $e \in M_{1} \cup M_{2}$, then $w^{\prime}(e)=\Delta^{\prime}$.

It is clear that the instance $I^{\prime}=\left(G^{\prime}, w^{\prime}\right)$ verifies the required hypothesis. We claim that $\operatorname{opt}(I) \leq \frac{\Delta(\Delta+1)}{2}$ iff $\operatorname{opt}\left(I^{\prime}\right) \leq \frac{\Delta^{\prime}\left(\Delta^{\prime}+1\right)}{2}$. 
If $\operatorname{opt}(I) \leq \frac{\Delta(\Delta+1)}{2}$, then by duplicating this edge coloring to $G_{i}$ and by adding a new color to $M_{1} \cup M_{2}$, we obtain an edge coloring of $G^{\prime}$ and $\operatorname{opt}\left(I^{\prime}\right) \leq \operatorname{opt}(I)+\Delta^{\prime} \leq \frac{\Delta^{\prime}\left(\Delta^{\prime}+1\right)}{2}$.

Conversely, assume that $\operatorname{opt}\left(I^{\prime}\right) \leq \frac{\Delta^{\prime}\left(\Delta^{\prime}+1\right)}{2}$. Then the edges of $M_{1} \cup M_{2}$ have the same color. Otherwise, $\operatorname{opt}\left(I^{\prime}\right) \geq \Delta^{\prime}+\Delta^{\prime}+\sum_{i=1}^{\Delta^{\prime}-2} i>\frac{\Delta^{\prime}\left(\Delta^{\prime}+1\right)}{2}$ since in $I^{\prime}$ there are $\Delta^{\prime}$ edges $e_{1}, \ldots, e_{\Delta^{\prime}}$ with $w^{\prime}\left(e_{i}\right)=i$ adjacent to the same vertex. Thus, the restriction of this solution to $G_{1}$ is an edge coloring verifying $\operatorname{opt}(I) \leq \operatorname{opt}\left(I^{\prime}\right)-\Delta^{\prime}$.

\subsection{Partial $k$-Trees}

A $k$-tree is a graph that can be reduced to a clique of size $k$ by deleting iteratively some vertices the neighborhood of which is a clique of size $k$. A partial $k$-tree is a subgraph of a $k$-tree. There are several characterizations of partial $k$-trees. One of them is the following: $G$ is a partial $k$-tree iff $G$ is a subgraph of a chordal graph $G^{\prime}$ with a clique number equal to $k+1$ (i.e., $\omega\left(G^{\prime}\right)=k+1$ ). $k$-trees are (polynomially) $k+1$-colorable, and we can get a $\rho_{k+1}$-approximation, but we can improve this result.

Let us define the List Coloring problem, where we want to answer the following question: given a graph $G=(V, E)$ with, for any $v \in V$, a set $L(v)$ of admissible colors, does there exist a (proper) coloring of $G$ with colors from $L(V)=\cup_{v \in V} L(v)$ such that any vertex $v$ is colored with a color from $L(v)$ ? The complexity of List Coloring has been studied in $[13,11]$.

Theorem 6 If $\mathcal{G}$ is a class of $t$-colorable graphs (where $t$ is a constant) where List Coloring is polynomial, then Weighted Coloring admits a PTAS in $\mathcal{G}$.

Proof. Let $\mathcal{G}$ such a class of graphs, a graph $G \in \mathcal{G}$ and $\varepsilon>0$. Let $k=\left\lceil(t-1)\left(1+\frac{1}{\varepsilon}\right)\right\rceil$.

Consider the following algorithm. For any $k^{\prime} \leq k$ :

- consider any $k^{\prime}$-tuple $\left(x_{1}, \cdots, x_{k^{\prime}}\right) \in W^{k^{\prime}}$;

- find a $k^{\prime}$-coloring $\left(S_{1}, \cdots, S_{k^{\prime}}\right)$ of $G$ such that $w\left(S_{i}\right) \leq x_{i}, i=1, \cdots, k^{\prime}$, if such a coloring exists;

- output the best coloring among those found in the previous step.

To achieve the second step, we use the fact that List Coloring is polynomial in $\mathcal{G}$. Indeed, given $\left(x_{1}, \cdots, x_{k^{\prime}}\right) \in W^{k^{\prime}}$, we can define an instance of List Coloring on $G: v_{i}$ can be colored with color $S_{j}$ for all $j \in\left\{1, \cdots, k^{\prime}\right\}$ such that $w\left(v_{i}\right) \leq x_{j}$. One can easily see that a coloring is valid for this instance of List Coloring, if and only if this coloring is such that $w\left(S_{i}\right) \leq x_{i}, i=1, \cdots, k^{\prime}$. 
We claim that the solution computed by this algorithm is $1+\varepsilon$-approximate, for any $\varepsilon>0$.

Indeed, consider an optimal solution $\mathcal{S}^{*}=\left(S_{1}^{*}, \cdots, S_{l}^{*}\right)$, with $w\left(S_{1}^{*}\right) \geq \cdots \geq w\left(S_{l}^{*}\right)$. If $l \leq k$, then we found, by our exhaustive search, a coloring $\left(S_{1}, \cdots, S_{l}\right)$ such that $w\left(S_{i}\right) \leq w\left(S_{i}^{*}\right)$ for all $i$, hence an optimal solution.

If $l>k$, then consider the $k$-tuple $\left(w_{1}, \cdots, w_{k}\right)$ where $w_{i}=w\left(S_{i}^{*}\right)$ for $i \leq k+1-t$ and $w_{i}=w\left(S_{k+1-t}^{*}\right)$ for $i \geq k+1-t$. If we consider the $k-t$ colors $S_{i}^{*}, i=1, \cdots, k-$ $t$, and any $t$-coloring $S_{j}, j=k+1-t, \cdots, k$ of the remaining vertices (the graph is polynomially $t$-colorable), then $w\left(S_{j}\right) \leq w\left(S_{k+1-t}^{*}\right)$ for $j=k+1-t, \cdots, k$. So, the algorithm finds a coloring for this particular tuple $\left(w_{1}, \cdots, w_{k}\right)$. Consequently, the solution $\mathcal{S}$ given by the algorithm is such that:

$$
\begin{aligned}
\frac{w(\mathcal{S})}{w\left(\mathcal{S}^{*}\right)} & \leq \frac{\sum_{i=1}^{k+1-t} w\left(S_{i}^{*}\right)+(t-1) w\left(S_{k+1-t}^{*}\right)}{\sum_{i=1}^{l} w\left(S_{i}^{*}\right)} \\
& \leq 1+\frac{(t-1) w\left(S_{k+1-t}^{*}\right)}{\sum_{i=1}^{k+1-t} w\left(S_{i}^{*}\right)} \leq 1+\frac{t-1}{k+1-t} \leq 1+\varepsilon
\end{aligned}
$$

Since we use less than $\left|W^{k+1}\right| \leq n^{k+1}$ times the algorithm for List Coloring as a subroutine, our algorithm is polynomial, hence we get the expected result.

Since List Coloring is polynomial in partial $k$-trees ([11]), then we have the following corollary:

Corollary 3 Weighted Coloring admits a PTAS in partial $k$-trees (hence, in particular, in trees).

Although we have proposed an approximation scheme in partial $k$-trees, the complexity of the Weighted Coloring problem remains open for these graphs.

We now focus ourselves on the case where the input graph is a chain, or a collection of chains. Guan and Zhu proposed in [8] a polynomial time algorithm with complexity $O\left(n^{4}\right)$ to solve (optimally) Weighted Coloring in chains (as a particular case of a more general result). We can improve this result in the following way:

Theorem 7 Weighted Coloring is polynomially solvable in $O(n|W|) \leq O\left(n^{2}\right)$ in chains.

Proof. Consider a graph $G$ which is a set of $k$ disjoint chains $C_{1}, \cdots, C_{k}$. Note that $\Delta(G) \leq 2$ hence any optimal Weighted Coloring has at most 3 colors. As we have seen previously, the best 2-coloring is easily computable (in $O(n)$ ). To compute an optimal 3coloring, we compute, for any $w \in W$, the smallest number $n_{w} \leq w$ for which there exists a 3-coloring $\left(S_{1}, S_{2}, S_{3}\right)$ with $w\left(S_{1}\right)=w_{\max }, w\left(S_{2}\right) \leq w$ and $w\left(S_{3}\right) \leq n_{w}\left(n_{w}=\infty\right.$ if such a coloring does not exist). Remark first that every vertex $v$ with weight $w(v)>w$ 
must receive color 1 . Consider now two consecutive such vertices $v_{i}, v_{j}$ in a chain $C_{l}$. If there exists an odd number of vertices between $v_{i}$ and $v_{j}$, we can color these vertices with colors 1 and 2. Otherwise, one must use 3 colors, and one can do it by coloring with color 3 only the lightest vertex $v_{i j}$ between $v_{i}$ and $v_{j}$. Hence, $n_{w}$ is the heaviest among these vertices $v_{i j}$. Given $w \in W$, we can find $n_{w}$ (and the corresponding coloring) in $O(n)$, hence the result follows.

\section{Note on related works}

During the time between submission and the current version, we have learnt that some results obtained here also appear in papers $[14,15]$. In these papers, the authors deal with the same problem that they call maximum coloring. In [14], the authors proved that this problem is NP-complete in interval graphs and gave constant polynomial approximation. However, the proof used for the NP-completeness is completely different. In [15], the authors proposed a PTAS for the case of trees. Moreover, they also provide a $(4-3 / k)$-approximation algorithm in hereditary classes of $k$-colorable graphs where the usual coloring problem is polynomial. In particular, this gives a $(4-3 / \Delta)$-approximation algorithm for line graphs of bipartite graphs with maximum degree $\Delta$.

\section{References}

[1] C. Berge. Graphs and Hypergraphs. North Holland, Amsterdam, 1973.

[2] M. Boudhar and G. Finke. Scheduling on a batch machine with job compatibilities. Special issue ORBEL-14: Emerging challenges in operations research (Mons, 2000). Belg. J. Oper. Res. Statist. Comput. Sci., 40(1-2):69-80, 2000.

[3] D. de Werra, M. Demange, B. Escoffier, J. Monnot and V.Th. Paschos. Weighted coloring on planar, bipartite and split graphs: complexity and improved approximation. Proc. ISAAC'04, LNCS 3341, 896-907, 2004.

[4] D. de Werra, M. Demange, J. Monnot and V.Th. Paschos. Weighted node coloring: when stable sets are expensive. Proc. WG'02, LNCS 2573:114-125, 2002.

[5] G. Finke, V. Jost, M. Queyranne, A. Sebö and X. Zhu. Batch Processing With Interval Graph Compatibilities Between Tasks. Cahiers du laboratoire Leibniz, 108, 2004, (available at http://www-leibniz.imag.fr/NEWLEIBNIZ/LesCahiers/index.xhtml.)

[6] M.R. Garey, D.S. Johnson, G.L. Miller and C.H. Papadimitriou. The complexity of coloring circular arcs and chords. SIAM J. Algebraic Dicrete Methods, 1:216-222, 1980 . 
[7] M. R. Garey and D. S. Johnson. Computers and intractability. a guide to the theory of NP-completeness. CA, Freeman, 1979.

[8] D. J. Guan and X. Zhu. A Coloring Problem for Weighted Graphs. Inf. Process. Lett., 61(2):77-81 1997.

[9] M. C. Golumbic. Algorithmic Graph Theory and Perfect Graphs. Academic Press, New York, 1980.

[10] H. Grotzsch, Ein dreifarbensatz fur dreikreisfreie netze auf der kugel, Wiss. Z. Martin Luther Univ. Halle-Wittenberg, Math. Naturwiss Reihe 8 (1959), 109-120.

[11] K. Jansen and P. Scheffler: Generalized coloring for tree-like graphs. Discrete Applied Mathematics, 75:135-155, 1997.

[12] D. König. Über graphen und iher anwendung auf determinantentheorie und mengenlehre. Math. Ann., 77:453-465, 1916.

[13] J. Kratochvíl and Z. Tuza. Algorithmic complexity of list colorings. Discrete Applied Mathematics, 50(3): 297-302, 1994.

[14] S. V. Pemmaraju, R. Raman and K. R. Varadarajan. Buffer minimization using maxcoloring. SODA 562-571, 2004.

[15] S. V. Pemmaraju and R. Raman. Approximation algorithms for the max-coloring. ICALP, (to appear) 2005. 